\title{
Flop of Electric Polarization Driven by the Flop of the Mn Spin Cycloid in Multiferroic $\mathrm{TbMnO}_{3}$
}

\author{
N. Aliouane, ${ }^{1,2}$ K. Schmalzl, ${ }^{3}$ D. Senff, ${ }^{4}$ A. Maljuk, ${ }^{1}$ K. Prokeš, ${ }^{1}$ M. Braden, ${ }^{4}$ and D. N. Argyriou ${ }^{1, *}$ \\ ${ }^{1}$ Helmholtz-Zentrum Berlin für Materialen und Energy, Glienicker Straße 100, D-14109 Berlin, Germany \\ ${ }^{2}$ Institute For Energy Technology, Post Office Box 40, NO-2027 Kjeller, Norway \\ ${ }^{3}$ Institut für Festkörperforschung, Forschungszentrum Jülich GmbH, JCNS at ILL, 38042 Grenoble Cedex 9, France \\ ${ }^{4}$ II. Physikalisches Institut, Universität zu Köln, Zülpicher Straße 77, D-50937 Köln, Germany
}

(Received 11 February 2009; published 20 May 2009)

\begin{abstract}
Using in-field single-crystal neutron diffraction, we have determined the magnetic structure of $\mathrm{TbMnO}_{3}$ in the high field $\mathbf{P} \| a$ phase. We unambiguously establish that the ferroelectric polarization arises from a cycloidal Mn spin ordering, with spins rotating in the $a b$ plane. Our results demonstrate directly that the flop of the ferroelectric polarization in $\mathrm{TbMnO}_{3}$ with applied magnetic field is caused from the flop of the Mn cycloidal plane.
\end{abstract}

DOI: 10.1103/PhysRevLett.102.207205

An exciting feature of novel magnetoelectric materials or multiferroics [1] is the flop of the ferroelectric polarization by the application of magnetic fields [2,3]. This effect realized in perovskites $R \mathrm{MnO}_{3}, R=\mathrm{Gd}, \mathrm{Tb}$, and $\mathrm{Dy}$, as well as in the system $\mathrm{Eu}_{1-x} \mathrm{Y}_{x} \mathrm{MnO}_{3}$, allows for the control of ferroelectricity by magnetic fields [1-3] and is relevant for new spin based device applications. Following its first observation in the $R \mathrm{MnO}_{3}$ materials this effect has been established in a wide variety of multiferroic materials such as spinel chromate $\mathrm{CoCr}_{2} \mathrm{O}_{4}$ [4], spin-chain cuprate $\mathrm{LiCu}_{2} \mathrm{O}_{2}$ [5], and huebnerite $\mathrm{MnWO}_{4}$ [6,7]. In these materials, the antisymmetric Dzyaloshinski-Moriya (DM) interaction [8,9] between two spins, $S_{i}, S_{i+1}$ separated by $\mathbf{r}_{i, i+1}$, leads to a cycloidal magnetic order shown in Fig. 1(a). This magnetic ordering provides for a natural coupling between magnetism and ferroelectricity with the spontaneous ferroelectric polarization given by $\mathbf{P}_{\mathbf{s}} \sim$ $\mathbf{r}_{i, i+1} \times\left(\mathbf{S}_{i} \times \mathbf{S}_{i+1}\right)[1,10,11]$ in these multiferroic materials [2-7]. The rotation of the ferroelectric polarization is proposed to arise from the rotation of the spin cycloid around $\mathbf{r}_{i, i+1}$ as a magnetic field is applied [10]. In this Letter we present a determination of the magnetic structure of one of the prototypical multiferroic manganite perovskites $\mathrm{TbMnO}_{3}$ that exhibits ferroelectricity due to the DM interaction and show that the discontinuous flop of the ferroelectric polarization arises directly from the flop of the Mn spin cycloid. Our finding validates this model and describes the change in the direction of the polarization as a function of applied field in multiferroic perovskite manganites and other similar cycloidal multiferroic compounds in terms of a change in the magnetic symmetry.

The behavior of $\mathrm{TbMnO}_{3}$ exemplifies that of many multiferroics whose magnetoelectric properties arise from the DM interaction. Here on cooling below $T_{N}=$ $41 \mathrm{~K} \mathrm{Mn}$ spins order with an incommensurate magnetic wave vector $\boldsymbol{\tau} \sim 0.275 \mathbf{b}^{*}$ and point along the $b$ axis [1,2]. On further cooling below $T_{S}=28 \mathrm{~K}$, a $c$ axis component of the Mn moment orders with a phase shift of $\pi / 2$ with respect to the $b$ component so as to form a cycloidal
PACS numbers: 75.25.+z, 61.05.F-, 75.10.-b, 75.30.Et

structure where Mn spins rotate within the $b c$ plane and around the $a$ axis as shown in Fig. 1(a) [1]. The axis of spin rotation defines the DM interaction, $\mathbf{S}_{i} \times \mathbf{S}_{i+1}$, while the distance $\mathbf{r}_{i, i+1}$ is parallel to $\boldsymbol{\tau}$. For this type of spin order, inversion symmetry is broken, yielding for $R=\mathrm{Tb}$ and Dy a ferroelectric polarization along the $c$ axis as indeed is observed $\left(\mathbf{P}_{\mathbf{s}} \| \mathbf{c} \sim \mathbf{a} \times \mathbf{b}\right)[1,2]$.

The application of magnetic field results in the flop of the polarization from the $c$ to the $a$ axis and highlights a novel control of one ferroic property by another [2]. It has been assumed that this change in the direction of the polarization reflects the flop of the Mn spin cycloid, implying that the antisymmetric DM interaction continues to be responsible for the polarization in high magnetic fields $[10,12]$. While it is tempting to assume that the flop in the ferroelectric polarization arises from a flop in the Mn spin spiral, so far there is no experimental evidence for this. The fact that in this $\mathbf{P} \| a$ phase Mn spins order commensurately with $\boldsymbol{\tau}=\frac{1}{4} \mathbf{b}^{*}[13,14]$ renders the cycloidal flop model even more complex. For such a commensurate spin structure it has been proposed that spin frustration and superexchange induce lattice distortions that break inversion symmetry thereby generating the observed direction of the polarization at high field $[13,15,16]$. This exchangestriction model can be applied only to a commensurate order, ruling out its validity for $R=$ Dy [17]. The observation that infrared electromagnon signals are always polarized along the $a$ axis irrespective of if $\mathbf{P}_{s}$ is parallel to the $a$ or $c$ axis further adds to the debate of the high field magnetic phases in these manganites [18]. A cycloidal Mn magnetic ordering that yields $\mathbf{P} \| a$ in zero field has been observed for $\mathrm{Gd}_{0.7} \mathrm{~Tb}_{0.3} \mathrm{MnO}_{3}$ [19]; however, there is no direct evidence that the magnetic field drives the $\mathrm{Mn}$ cycloid to flop and yield a $\mathbf{P} \| a$ polarization. It is therefore pressing to establish an accurate model of the high field magnetic structure of these multiferroic manganites.

To establish the high magnetic-field structure in the flopped $\mathbf{P} \| a$ phase, we have chosen to investigate the multiferroic $\mathrm{TbMnO}_{3}$, as the smaller neutron absorption 


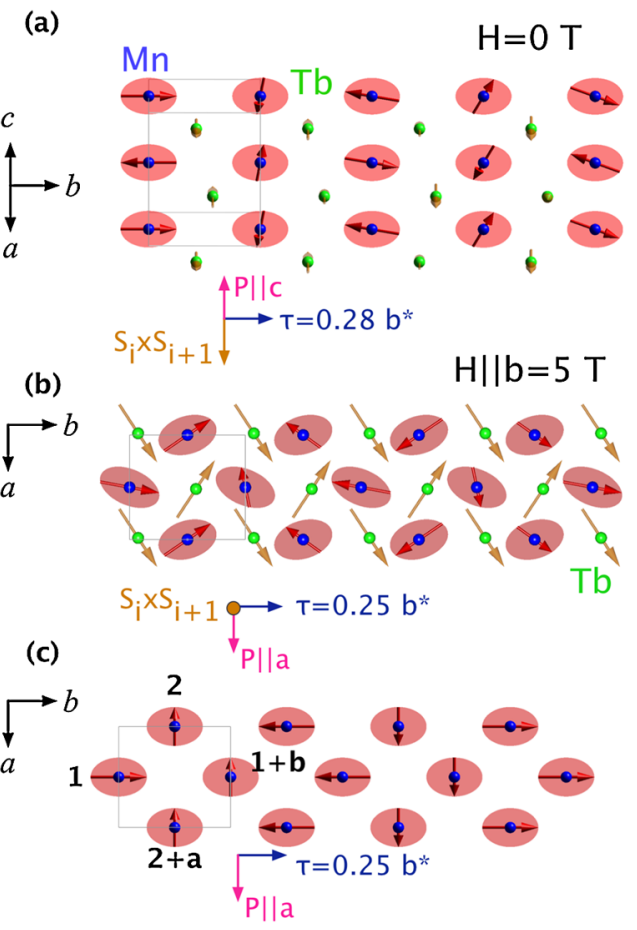

FIG. 1 (color online). (a, b) Illustrations of the two cycloidal Mn magnetic structures proposed to cause ferroelectricity in $\mathrm{TbMnO}_{3}$. In both cases the magnetic propagation vector $\tau$ is parallel to the $b$ axis. In zero field [panel (a)] cycloidal order, Mn spins rotate around the $a$ axis $\left(S_{i} \times S_{i+1}\right)$ and rotate wholly within the $b c$ plane. The ferroelectric polarization via the antisymmetric DM interaction is produced along the $c$ axis. The high field magnetic structure determined in this work is shown in panel (b); it yields a $\mathbf{P} \| a$ ferroelectric polarization that arises from an $a b$ cycloid where Mn spins rotate around the $c$ axis. In both panels we also show the magnetic ordering of $\mathrm{Tb}$ spins. In the zero field case (a), the magnetic propagation vectors of $\mathrm{Tb}$ and $\mathrm{Mn}$ spins are clamped, and Tb spins point along the $a$ axis forming a spin-density wave [1]. The canted antiferromagnetic ordering of Tb spins for $H \| b=5 \mathrm{~T}$ determined in this work is shown in panel (b). (c) Here we depict an anharmonic $a b$ spiral where the phase difference between spins 1 and 2 is $\omega=\pi / 2$. In such a case the angle between spins 1 and 2 is different from that between 2 and $1+b$ yielding an alternating scalar product along the $b$ axis. Amplitudes of $\mathrm{Tb}$ and $\mathrm{Mn}$ spins are not to scale.

cross section of $\mathrm{Tb}$ facilitates neutron experiments in sharp contrast to the much higher cross sections of Dy, Eu, or Gd. $\mathrm{TbMnO}_{3}$ single crystals were obtained by recrystalizing a ceramic rod under Ar atmosphere using an optical floatingzone furnace. The field and temperature dependence of the magnetic propagation wave vector was measured on the E4 double axis neutron diffractometer at the BENSC facility of Helmholtz-Zentrum Berlin using a neutron wavelength of $\lambda=2.45 \AA$ and a $\lambda / 2$ filter placed in the incident beam. Here the magnetic field was applied horizontally along the $b$ axis using the HM1 superconducting cryomagnet. Because of the limited view of the sample in this magnet, analysis of the magnetic structure was not possible. To overcome this problem we conducted measurements using the D23 neutron single-crystal diffractometer installed at the Institut Laue-Langevin with $\lambda=1.281 \AA$, which is equipped with a lifting detector that allows measurements of Bragg reflections above and below the scattering plane. The magnetic field was applied using the $6 \mathrm{~T}$ vertical field superconducting magnet with an asymmetric vertical opening angle of $-5^{\circ} /+10^{\circ}$. For these measurements a single crystal of $\mathrm{TbMnO}_{3}$ was cut into a parallelepiped with dimension $3.0 \times 2.9 \times 3.7 \mathrm{~mm}$ with each face perpendicular to one crystallographic direction. The crystal was oriented with the $b$ axis parallel to the field. This geometry allowed us to measure reflections with $k$ from -1.8 to 0.25 . In total, 138 independent nuclear Bragg reflections consisting of 310 individual reflections were collected at $H=0 T$ and $T=8.5 \mathrm{~K}$ within a range of $0.07 \leqq \sin \theta / \lambda \leqq 0.69 \AA^{-1}$. At $8.5 \mathrm{~K}$ the magnetic field was applied along the $b$ axis to $5 \mathrm{~T}$. In these conditions 140 commensurate $(\boldsymbol{\tau}=0)$ independent Bragg reflections consisting of 160 individual reflections were measured along with 64 independent reflections with $\boldsymbol{\tau}=\frac{1}{4} \mathbf{b}^{*}$. Analytical absorption corrections for each reflection were made by the XTAL suite of programs, while analysis of the magnetic intensities was performed with the FULLPROF code.

In Fig. 2(a) we show measurements of the $(0, \epsilon, 1)$ reflection as a function of temperature with $H \| b=5 \mathrm{~T}$ measured on the $\mathrm{E} 4$ diffractometer. The data show that $\mathrm{Mn}$ spin ordering is first observed at $T_{N}=41 \mathrm{~K}$ with the wave number $\epsilon$ decreasing on cooling. At $T_{c}=11 \mathrm{~K}, \epsilon$ changes

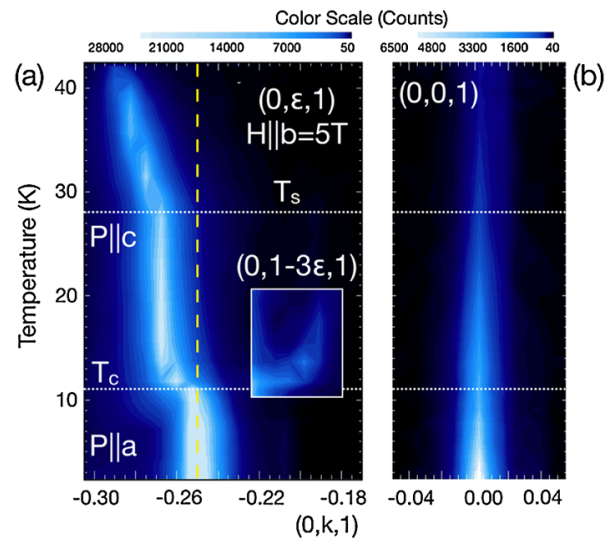

FIG. 2 (color online). Single-crystal neutron diffraction measurements from the E4 diffractometer. Here we show scans along $(0, k, 1)$ in reciprocal space as a function of temperature measured in $H \| b=5 \mathrm{~T}$. The intensity of these scans is plotted in color coding with corresponding scales above each panel. (a) Portion of the data showing the temperature dependence of the A mode reflection $(0, \epsilon, 1)$. Here the wave number $\epsilon$ varies from 0.282 at $T_{N}$ to 0.266 at $15 \mathrm{~K}$ before it locks discontinuously to the commensurate value of $\frac{1}{4}$ below $11 \mathrm{~K}$. The weak third harmonic reflection was also observed in these scans to disappear at this transition. These data are shown in an enhanced color scale on the same panel and in the same location in the $T$ - $Q$ map. (b) Temperature evolution of the Pbnm forbidden reflection $(0,0,1)$. 
discontinuously to yield a commensurate wave vector of $\boldsymbol{\tau}=\frac{1}{4} \mathbf{b}^{*}[13,14,20]$. Published polarization data show that a $\mathbf{P} \| c$ state develops below $T_{S}$ while the polarization flops to $\mathbf{P} \| a$ at $T_{c}$, coinciding with the transition to the commensurate magnetic phase [21]. In the same data we observe that the third harmonic reflection $(0,1-3 \epsilon, 1)$ rapidly changes its position and disappears also at $T_{c}$ [Fig. 2(a)]. On cooling below $T_{N}$ we find an enhancement in the intensity of several nuclear reflections and the appearance of forbidden reflections such as the $(0,0,1)$ shown in Fig. 2(b). As we discuss below, these effects arise from the ordering of $\mathrm{Tb}$ spins with magnetic propagation vector $\tau^{\mathrm{Tb}}=0$.

To determine the magnetic structure of $\mathrm{TbMnO}_{3}$ in the P || $a$ state, we utilized the D23 diffractometer. Here the sample was cooled in zero field to $8.5 \mathrm{~K}$, and then a $H \| b=5 \mathrm{~T}$ was applied so as to enter the commensurate P $\|$ a phase that was confirmed by measurements of the magnetic wave vector. At this field and temperature we find that the most intense magnetic reflections with wave number $\epsilon=\frac{1}{4}$ have extinction condition $h+k=$ even, $l=$ odd (A mode) [22], while reflections with $h+k=$ odd, $l=$ odd (G mode) were considerably weaker. For space group $P b n m$ and wave vector $\tau \leq \frac{1}{4} \mathbf{b}^{*}$ there are four irreducible representations (irreps) $\Gamma$ of the magnetic symmetry for the $\mathrm{Mn}$ ion $[23,24]$. The A mode reflections are contained only in irreps $\Gamma_{1}, \Gamma_{2}$, and $\Gamma_{3}$. Analysis of the data using only A mode reflections and a single irrep did not result in a satisfactory fit to the data. This leads us to consider combinations of representations. Among the possible combinations only a model using $\Gamma_{1} \otimes \Gamma_{3}$ produced a satisfactory result with agreement index $R=7.9 \%$ and weighted $R_{w}=8.5 \%$ [25]. This coupled irrep has the form of $\left(A_{x}, G_{y}, C_{z}\right) \otimes\left(G_{x}, A_{y}, F_{z}\right)$. Our measurements showed that $\mathrm{F}$ and $\mathrm{C}$ modes were at the very limit of detection, indicating that the $\mathrm{Mn}$ moment is essentially contained within the $a b$ plane. Reflections from these modes were not included in the final refinements [26]. Our analysis yielded a magnetic structure for Mn given by the moment in $\mu_{B} / \mathrm{Mn}: m_{1}=(2.83(12), 0.51(4), 0)$ and $m_{3}=(0.55(4), 3.79(7), 0)$, where the subscript denotes the irreducible representation. The phase between the two irreps is also a variable parameter found to be $0.474(12) \pi$, a value close to $\frac{\pi}{2}$ expected for a cycloid [27]. The values of the Mn spins in this commensurate cycloidal structure along the $a$ and $b$ axis are given in Fig. 3 for the $z=0$ and $z=\frac{1}{2}$ layers.

The magnetic structure indicated by this model is dominated by the $\mathrm{A}$ modes of these two irreps $\left(A_{x}\right.$ and $\left.A_{y}\right)$ producing an elliptical cycloid, shown in Fig. 1(b). Here the Mn cycloid is contained within the $a b$ plane, and Mn spins rotate around the $c$ axis, consistent with the direction of the ferroelectric polarization along the $a$ axis $(\mathbf{P} \sim \mathbf{b} \times$ c). The anisotropy of the Mn spin cycloid in both low field $b c$ and high field $a b$ configurations appears to be very similar. In both cases the $A_{y}$ mode possesses the higher moment (3.9 and $3.79 \mu_{B} / \mathrm{Mn}$, respectively), while the

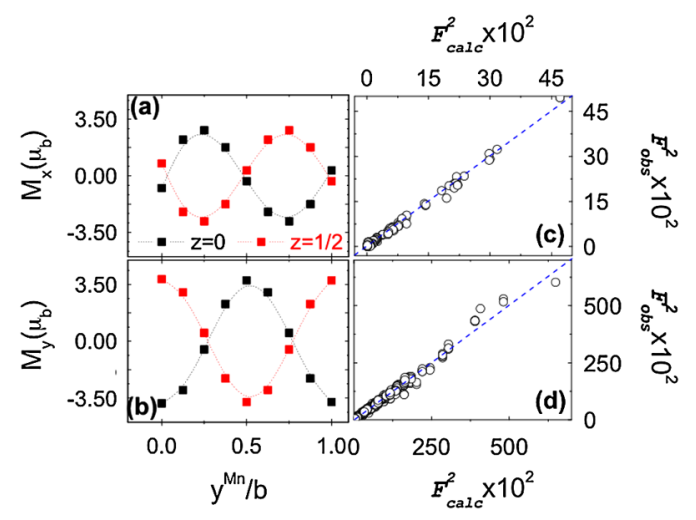

FIG. 3 (color online). Results of refinements of the magnetic structure at $8.5 \mathrm{~K}$ and $H \| b=5 \mathrm{~T}$. In panels (a) and (b) we show the variation of the $M_{x}$ and $M_{y}$ components of the Mn spins for the ions located at $z=0$ (black squares) and $z=\frac{1}{2}$ [gray (red) squares] along the magnetic propagation vector. Note that the components along $a$ and $b$ are out of phase so as to yield a cycloidal ordering shown in Fig. 1. In panels (c) and (d) we show the comparison between observed and calculated magnetic structure factors $\left(F^{2}\right)$ for the analysis of the Mn $\tau=\frac{1}{4}$ reflections and nuclear plus Tb magnetic reflections, respectively. Since the $\mathrm{Tb}$ magnetic propagation vector is $\tau=0$, the nuclear and magnetic reflections are not separated.

components orthogonal to this mode are smaller and of the same magnitude $\left(2.8 \mu_{B} / \mathrm{Mn}\right)$. The deviation of the phase shift between the two irreps away from the ideal value produces an angle between spins of $85^{\circ}$. Despite this, $\mathbf{S}_{\mathbf{i}} \times \mathbf{S}_{\mathbf{i}+\mathbf{1}}$ remains parallel to the $c$ axis and should not influence the magnitude of $\mathbf{P}_{S}$ significantly. Finally for the Mn ordering we find that the $G_{x}$ and $G_{y}$ modes are active with amplitudes of $\sim 0.5 \mu_{B} / \mathrm{Mn}$ and its net effect on the overall cycloidal order that produces a polarization along the $a$ axis is also relatively small.

We now turn our attention to the Tb spin ordering. We find that in the $\mathbf{P} \| a$ phase Tb spins order commensurately with the underlying primitive lattice (i.e., $\tau^{\mathrm{Tb}}=0$ ). Analysis of the commensurate Pbnm reflections clearly showed additional intensity that can be modeled by the $\mathrm{Tb}$ magnetic order. The best fit to the measured data was obtained for a ferromagnetic alignment of $\mathrm{Tb}$ spins along the $b$ axis and antiferromagnetic coupling between nearestneighbor $\mathrm{Tb}$ spins along the [110] direction [Fig. 1(b)]. Analysis of this structure yields a total $\mathrm{Tb}$ moment of $7.24(7) \mu_{B}$, with an antiferromagnetic component of $6.07(9) \mu_{B}$ along the $a$ axis and a ferromagnetic component of 3.92(6) $\mu_{B}$ along the $b$ axis. The ferromagnetic ordering along the $b$ axis is indeed evident in magnetization measurements under similar conditions [21].

The work we present here unambiguously proves that the commensurate $\mathbf{P} \| a$ phase in $\mathrm{TbMnO}_{3}$ coincides with an $a b \mathrm{Mn}$ spin cycloid for $H \| b=5 \mathrm{~T}$. The antisymmetric $\mathrm{DM}$ interaction in this case does yield a ferroelectric polarization along the $a$ axis as indeed is observed $\left[\mathbf{P}_{\mathbf{s}} \| \mathbf{a}=\right.$ $\boldsymbol{\tau} \times\left(\mathbf{S}_{i} \times \mathbf{S}_{i+1}\right)=\mathbf{c} \times \mathbf{b}$ ]. We may thus identify the in- 
verse $\mathrm{DM}$ interaction as the main mechanism for the magnetic-field induced flop of ferroelectric polarization.

In a perfect cycloidal magnetic arrangement the exchange mechanism proposed in Refs. [15,16] does not yield any ferroelectric polarization, as the scalar product $\left(\mathbf{S}_{i} \cdot \mathbf{S}_{i+1}\right)$ of neighboring spins is everywhere the same. This still holds for the commensurate perfectly circular spiral. In the case of an elliptical commensurate cycloid the exchange mechanism does cause ferroelectric polarization as the scalar product varies along the modulation. In our case with a modulation of four orthorhombic lattice distances the mechanism of $[15,16]$ may thus yield a finite polarization along the $a$ direction which, however, should still be small due to the only minor deviation from a perfect circular cycloid. The exchange mechanism may gain further importance in the case of a very anharmonic cycloid. In the extreme anharmonic arrangement, where spins point either along the $b$ or $a$ directions yielding the sequence along the $b$ direction shown in Fig. 1(c) (left, up, right, down), there will be a very effective exchange-striction mechanism as the scalar product $\mathbf{S}_{i} \cdot \mathbf{S}_{i+1}$, between nearest-neighboring $\mathrm{Mn}$ ions, alternates along the $b$ axis [in the example of Fig. 1(c) the alternating angles are $90^{\circ}$ and $0^{\circ}$ along the $b$ direction]. In the current analysis we have performed, the phase difference between rows of cycloids propagating along the $b$ axis [e.g., in Fig. 1(c), rows starting at ions 1 and 2] is fixed by symmetry as $\psi=$ $2 \pi \tau / 2=45^{\circ}$ [24]. A deviation from this value will yield an anharmonic spiral, however such a case cannot be fully tested with the current data, and we cannot exclude a small degree of anharmonicity. In view of the current debate about the electromagnon it appears interesting to add that this exchange-striction mechanism will always yield ferroelectric polarization along the $a$ direction.

In conclusion our work demonstrates that the flop in the ferroelectric polarization observed in $\mathrm{TbMnO}_{3}$ arises from the flop of the Mn cycloidal plane from $b c$ to $a b$. The cycloidal magnetic structure we establish here for the high field $\mathbf{P} \| a$ phase does not posses a dominant ferroelectric mechanism based on exchange striction. The ordering of $\mathrm{Tb}$ spins in this high field phase is that of a canted antiferromagnet giving a significant ferromagnetic component along the $b$ axis. Our results validate that the flop of $\mathbf{P}_{s}$ in the multiferroic perovskite manganites arises from a flop of the Mn spin cycloid.

We acknowledge the assistance of L.C. Chapon and Juan Rodriguez-Carvajal with FP-STUDIO and FULLPROF, respectively. We thank M. Mostovoy and D. Khomskii for helpful discussions. We acknowledge support from the Deutsche Forschungsgemeinschaft under Contract No. AR 613/1-1 (D. N. A.) and through SFB 608 (D. S. and M. B.).

\footnotetext{
*argyriou@helmholtz-berlin.de
}

[1] S.-W. Cheong and M. Mostovoy, Nature Mater. 6, 13 (2007).

[2] T. Kimura, T. Goto, H. Shintani, K. Ishizaka, T. Arima, and Y. Tokura, Nature (London) 426, 55 (2003).

[3] T. Goto, T. Kimura, G. Lawes, A. P. Ramirez, and Y. Tokura, Phys. Rev. Lett. 92, 257201 (2004).

[4] Y. Yamasaki, S. Miyasaka, Y. Kaneko, J. P. He, T. Arima, and Y. Tokura, Phys. Rev. Lett. 96, 207204 (2006).

[5] S. Park, Y. J. Choi, C. L. Zhang, and S.-W. Cheong, Phys. Rev. Lett. 98057601 (2007).

[6] O. Heyer, N. Hollmann, I. Klassen, S. Jodlauk, L. Bohaty, P. Becker, J. A. Mydosh, T. Lorenz, and D. Khomskii, J. Phys. Condens. Matter 18, L471 (2006).

[7] K. Taniguchi, N. Abe, T. Takenobu, Y. Iwasa, and T. Arima, Phys. Rev. Lett. 97, 097203 (2006).

[8] I. Dzyaloshinsky, Sov. Phys. JETP 19, 960 (1960).

[9] T. Moriya, Phys. Rev. 120, 91 (1960).

[10] M. Mostovoy, Phys. Rev. Lett. 96, 067601 (2006).

[11] H. Katsura, N. Nagaosa, and A. V. Balatsky, Phys. Rev. Lett. 95, 057205 (2005).

[12] D. Senff, P. Link, N. Aliouane, D. N. Argyriou, and M. Braden, Phys. Rev. B 77, 174419 (2008).

[13] N. Aliouane, D. N. Argyriou, J. Strempfer, I. Zegkinoglou, S. Landsgesell, and M.v. Zimmermann, Phys. Rev. B 73, 020102(R) (2006).

[14] T. Arima, T. Goto, Y. Yamasaki, S. Miyasaka, K. Ishii, M. Tsubota, T. Inami, Y. Murakami, and Y. Tokura, Phys. Rev. B 72, 100102(R) (2005).

[15] I. A. Sergienko and E. Dagotto, Phys. Rev. B 73, 094434 (2006).

[16] I. A. Sergienko, C. Sen, and E. Dagotto, Phys. Rev. Lett. 97, 227204 (2006).

[17] J. Strempfer, B. Bohnenbuck, M. Mostovoy, N. Aliouane, D. N. Argyriou, F. Schrettle, J. Hemberger, A. Krimmel, and M. v. Zimmermann, Phys. Rev. B 75, 212402 (2007).

[18] N. Kida, Y. Ikebe, Y. Takahashi, J. P. He, Y. Kaneko, Y. Yamasaki, R. Shimano, T. Arima, N. Nagaosa, and Y. Tokura, Phys. Rev. B 78, 104414 (2008).

[19] Y. Yamasaki, H. Sagayama, N. Abe, T. Arima, K. Sasai, M. Matsuura, K. Hirota, D. Okuyama, Y. Noda, and Y. Tokura, Phys. Rev. Lett. 101, 097204 (2008).

[20] D. Senff, P. Link, K. Hradil, A. Hiess, L. P. Regnault, Y. Sidis, N. Aliouane, D. N. Argyriou, and M. Braden, Phys. Rev. Lett. 98, 137206 (2007).

[21] T. Kimura, G. Lawes, T. Goto, Y. Tokura, and A.P. Ramirez, Phys. Rev. B 71, 224425 (2005).

[22] $h, k, l$ are Miller indices at the center of the primitive Brillouin zone.

[23] E. F. Bertaut, J. Magn. Magn. Mater. 24, 267 (1981).

[24] H. W. Brinks, J. Rodríguez-Carvajal, H. Fjellvåg, A. Kjekshus, and B.C. Hauback, Phys. Rev. B 63, 094411 (2001).

[25] Here $R$ factors are based on $F^{2}$, where $F$ is the magnetic structure factor.

[26] Reflections condition for the $\mathrm{C}$ mode are $h+k=$ even, $l=$ even and for the $\mathrm{F}$ mode $h+k=$ odd, $l=$ even

[27] Our sensitivity to the phase difference was tested by fixing it to a value of $\pi / 2$ which leads to statistically significantly higher $R$ factors of $R\left(F^{2}\right)=8.4 \%$ and $R_{w}\left(F^{2}\right)=9 \%$. 